\title{
A Novel Broadband Electro-Absorption Modulator Based on Bandfillin in n-InGaAs: Design and Simulations
}

\author{
Jorn P. van Engelen ${ }^{(1)}$, Longfei Shen, Gunther Roelkens ${ }^{(1)}$, Member, IEEE, Yuqing Jiao ${ }^{(0)}$, Member, IEEE, \\ Meint K. Smit, Fellow, IEEE, and Jos J. G. M. van der Tol
}

\begin{abstract}
We propose and evaluate by simulation a novel membrane electro-absorption modulator heterogeneously integrated on silicon. The device is based on the electron-concentrationdependent absorption of highly doped n-InGaAs. It is predicted that the modulator can be operated over a wavelength range of more than $100 \mathrm{~nm}$ and provides a static extinction ratio of $7.2 \mathrm{~dB}$, an insertion loss of $4.4 \mathrm{~dB}$, a modulation speed above $50 \mathrm{~Gb} / \mathrm{s}$, and a power consumption of $53 \mathrm{fJ} / \mathrm{b}$. The modulator has a small footprint of $0.4 \times 80 \mathrm{um}^{2}$ (excluding contact pads) and operates on a CMOS compatible $1.5 \mathrm{~V}$ voltage swing.
\end{abstract}

Index Terms-Electro-absorption modulator, Burstein-Moss, bandfilling, InGaAs, Silicon, Indium phosphide membrane, Integrated photonics.

\section{INTRODUCTION}

$\mathbf{T}$ HE ever-increasing requirements on data transport networks create demand for high-density high-speed photonic integrated circuits [1]. The InP membrane on Si (IMOS) platform aims to satisfy this demand by combining high performance photonic integrated circuits with silicon electronics [2].

An essential building block in any photonic integration platform is the modulator. A small footprint and broadband switching are desired for e.g., Dense Wavelength Division Multiplexing (DWDM) applications. Integrated Electro-Absorption Modulators (EAMs) are often based on the Quantum-Confine Stark Effect (QCSE) or the Franz-Keldysh effect. QCSE modulators have a narrow optical bandwidth, while the Franz-Keldysh effect provides only limited absorption change leading to large insertion loss for large extinction ratio. Another approach is the use of phase modulation (e.g., Kerr effect) in an integrated Mach-Zehnder Interferometer (MZI) setup, but the two arms and splitters of the MZI make for a large footprint.

Manuscript received April 2, 2017; revised August 15, 2017; accepted October 16, 2017. Date of publication October 23, 2017; date of current version November 30, 2017. (Corresponding author: Jorn P. van Engelen).

J. P. van Engelen, L. Shen, Y. Jiao, M. K. Smit, and J. J. G. M. van der Tol are with the Photonic Integration Group, Institute for Photonic Integration (formerly the COBRA institute), Eindhoven University of Technology, Eindhoven 5612 AZ, The Netherlands (e-mail: j.p.v.engelen@tue.nl; 1.shen@tue.nl; y.jiao@tue.nl; m.k.smit@tue.nl; j.j.g.m.v.d.tol@tue.nl).

G. Roelkens is with the Photonics Research Group, Center for Nano- and Biophotonics, Ghent University_-IMEC, Gent 9000, Belgium (e-mail: gunther. roelkens@ugent.be).
Recent developements in EAMs that are heterogeneously integrated with silicon have shown improvements in modulation speed and optical bandwidth. State-of-the-art $50 \mathrm{GHz} \mathrm{Ge}-\mathrm{Si}$ EAMs [3], [4] based on the Franz-Keldysh effect demonstrate optical bandwidths of 10-20 nm. Silicon-based EAMs using Fermi-level variation in graphene show optical bandwidth in excess of $80 \mathrm{~nm}$ [5], but have low extinction ratio and limited modulation frequency due to high contact resistance to graphene. Another modulator that is currently being developed for the IMOS platform is predicted to have a high modulation speed [6] but has a large footprint due to the MZI structure.

Highly doped $\mathrm{n}$-InGaAs shows large changes in the absorption when the electron concentration is varied. The effect was firs measured and explained by Hahn in 1995 [7]. The change in absorption is due to a combination of band fillin and band-gap shrinkage in InGaAs, which causes electron-density-dependent absorption. The novel application of this effect in an EAM gives unique broadband behaviour compared to existing integrated modulators. Realization of a modulator based on this effect was previously not viable, but is now possible in the IMOS platform. This is because IMOS waveguides have high index contrast and therefore provide strong light-matter interaction [2]. The band fillin effect is inherently broadband and the modulator introduced here will have an optical bandwidth in excess of $100 \mathrm{~nm}$ covering the extended (E), short (S), conventional (C) and long (L) bands.

In this paper the modeling for the proposed modulator is introduced. First the electron-density-dependent absorption of $\mathrm{n}$-InGaAs, which is the basis of the modulator, is discussed. The proposed device structure and the trade-offs are discussed in Section III. The methodology of the semiconductor and optical simulations are given in Section IV and the results are discussed in Section V.

\section{BROADBAND ELECTRO-ABSORPTION MODULATION}

InGaAs lattice matched to InP has a bandgap of $0.74 \mathrm{eV}$, smaller than the photon energy in the $1.55 \mu \mathrm{m}$ telecommunication wavelength range. Therefore InGaAs is extensively used as the absorption material in $1.55 \mu \mathrm{m}$ photodetectors, where the photons have energies close to $0.8 \mathrm{eV}$. In n-type heavily-doped semiconductors however, a reduction of absorption near the bandgap is a well-known phenomenon, which is 
explained by the bandfillin effect [8] (also known as the Burstein-Moss effect). Energy states at the bottom of the conduction band are fille up by the high concentration of free electrons, so that photons require energies higher than the bandgap energy to excite an electron from the valence band to the conduction band. This leads to a higher apparent bandgap. While this effect also takes place for holes in the valence band, the effect is particularly strong for electrons owing to the lower density of states in the conduction band as compared to the valence band. InGaAs in particular has the lowest conduction band density of states in the GaInAsP/InP system, and therefore this material is used in the proposed EAM. By changing the electron density inside the $\mathrm{n}$-InGaAs layer, the optical absorption can be modulated.

Recently Shen et al. [9] used the bandfillin effect to explain the low-optical-loss in highly-doped n-InGaAs contact layers. In [9] a model by Weber [10] is used to predict the electronconcentration-dependent absorption in $\mathrm{n}$-InGaAs and includes three free-carrier induced effects: bandfilling bandgap shrinkage and free carrier absorption. The Urbach tail was neglected. To predict the absorption of $\mathrm{n}-\mathrm{InGaAs}$ the same model as in [9] is used to predict the performance of the proposed EAM. Selected equations from [9] and [10] are repeated here to explain the modeling.

The absorption is modeled as the combination of three carrierdependend effects: bandfillin (BF), bandgap shrinkage (BS), and free carrier absorption (FCA):

$$
\alpha_{\mathrm{m}}\left(E_{\mathrm{p}}, n\right)=\alpha_{\mathrm{BF}, \mathrm{BS}}\left(E_{\mathrm{p}}, n\right)+\alpha_{\mathrm{FCA}}(n) .
$$

The carriers are modeled firs as a cause for bandgap shrinkage, followed by their fillin of the band [9]:

$$
\alpha_{\mathrm{BF}, \mathrm{BS}}\left(E_{\mathrm{p}}, n\right)=\alpha_{\mathrm{IB}}\left(E_{\mathrm{p}}-\Delta E_{\mathrm{g}}(n)\right)\left[f\left(E_{\mathrm{v}}\right)-f\left(E_{\mathrm{c}}\right)\right],
$$

where $E_{\mathrm{p}}$ is the photon energy and $\Delta E_{\mathrm{g}}(n)$ the shrank bandgap energy that depends on the electron concentration $n\left(\Delta E_{\mathrm{g}}(n)\right.$ is modeled following[10, eq. (26)]). The absorption of the photon excites an electron from energy $E_{\mathrm{v}}$ in the valance band to energy $E_{\mathrm{c}}$ in the conduction band. For conciseness we discuss the case of only a single valance band but all results shown in this paper consider separate light hole and heavy hole bands that are summed up to a total $\alpha_{\mathrm{BF}, \mathrm{BS}}$ (as is done in [10, eq. (22)]). The probability of an occupied state in the valance band, and an empty state in the conduction band with a transition energy equal to the photon energy is given by $f\left(E_{\mathrm{v}}\right)-f\left(E_{\mathrm{c}}\right)$, with the Fermi-Dirac distribution:

$$
f\left(E_{\mathrm{v}, \mathrm{c}}\right)=\left[1+\exp \left(\frac{E_{\mathrm{v}, \mathrm{c}}-E_{\mathrm{F}}}{k_{\mathrm{B}} T}\right)\right]^{-1}
$$

The relation between $E_{\mathrm{v}}, E_{\mathrm{c}}$ and the photon energy follows from $\vec{k}$ conservation. Assuming parabolic bands, the relationship is given in [10, eq. (24)]. The Fermi level depends on the electron concentration and is define as:

$$
E_{\mathrm{F}}=k_{\mathrm{B}} T \cdot \mathcal{F}_{\frac{1}{2}}^{-1}\left(\frac{n}{N_{\mathrm{C}}}\right)+E_{\mathrm{C}}
$$

where $k_{\mathrm{B}}$ is the Boltzmann constant, temperature $T=300 \mathrm{~K}$, $\mathcal{F}_{\frac{1}{2}}^{-1}$ the inverse Fermi-Dirac integral of order $1 / 2, n$ the

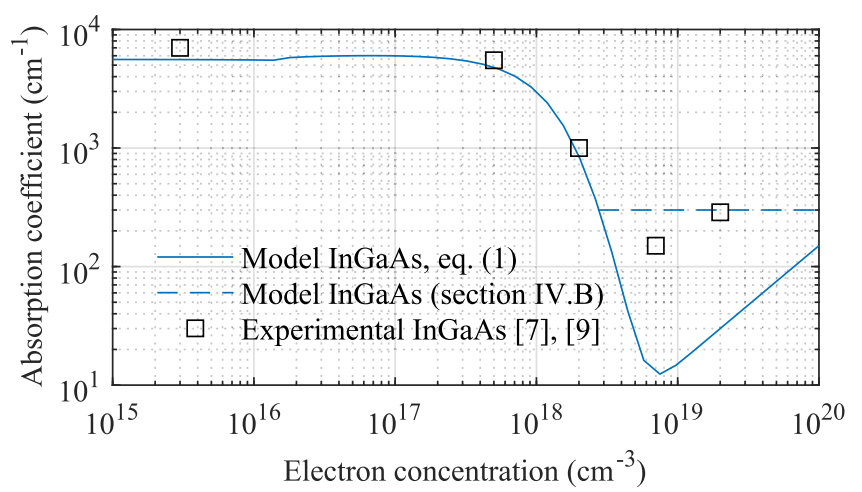

Fig. 1. Absorption coefficien at $1.55 \mu \mathrm{m}$ of n-type InGaAs as a function of electron concentration. The absorption shows a sharp drop above $10^{18} \mathrm{~cm}^{-3}$ due to the bandfillin effect.

electron concentration, $N_{\mathrm{C}}$ the conduction band density of states in InGaAs and $E_{\mathrm{C}}$ the conduction band edge. In the case of highly n-doped material $f\left(E_{\mathrm{v}}\right) \approx 1 . f\left(E_{\mathrm{c}}\right)$ increases with increasing electron concentration and approaches 1 , indicating a reduction of the absorption coefficien in (2).

The interband absorption coefficien is modeled by $[9$, eq. (1)]:

$$
\alpha_{\mathrm{IB}}(E)=\left\{\begin{array}{cl}
C E^{-1}\left(E-E_{\mathrm{g}}\right)^{\frac{1}{2}}, & E>E_{\mathrm{g}} ; \\
0, & E \leq E_{\mathrm{g}},
\end{array}\right.
$$

where $C=2.0 \cdot 10^{4} \mathrm{~cm}^{-1} \mathrm{eV}^{1 / 2}[10]$ is a material specifi constant and $E_{\mathrm{g}}$ the InGaAs bandgap energy.

The free-carrier-absorption $\alpha_{\mathrm{FCA}}$ depends on the electron concentration $n$ and is found to be nearly constant for wavelengths between 1 and $2 \mu \mathrm{m}$ [10]. We follow the model in [9, eq. (4)]:

$$
\alpha_{\mathrm{FCA}}(n)=A \cdot n, \quad A=1.5 \cdot 10^{-18} \mathrm{~cm}^{-2} .
$$

This completes the model for absorption in InGaAsP found in [10]. Here we apply the model to n-InGaAs with the same parameters as in [9] and present the absorption dependence of $\mathrm{n}$-InGaAs from (1) on electron density and photon energy.

A steep drop in the absorption coefficien is predicted for electron concentrations higher than $10^{18} \mathrm{~cm}^{-3}$ at a wavelength of $1.55 \mu \mathrm{m}$ (see Fig. 1). Experimental data from [7] shows reasonable agreement with this model. The rise of absorption beyond $1 \cdot 10^{19} \mathrm{~cm}^{-3}$ is due to free carrier absorption $\alpha_{\mathrm{FCA}}$.

The electron-density-dependent change in absorption of InGaAs is steepest around $3 \cdot 10^{18} \mathrm{~cm}^{-3}$. Variations of electron concentration around this doping level therefore have a large influenc on the absorption. The EAM proposed here varies the electron concentration by modulating the depletion depth into an n-InGaAs layer by using a p-i-n junction. This will be discussed further in Section III.

The absorption modulation becomes more broadband for increasing doping levels. This is because with higher doping levels the material becomes transparent for photons with higher energies (Fig. 2), while the material remains highly absorbing when depleted. Fig. 3 shows the material absorption difference between intrinsic InGaAs and doped n-InGaAs, 


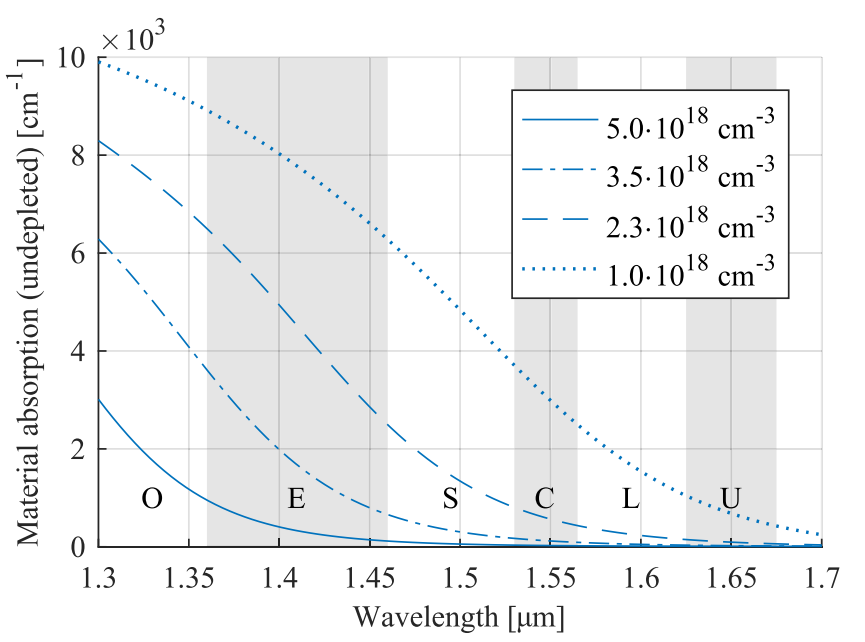

Fig. 2. Wavelength dependence of the absorption in n-InGaAs, with telecom bands indicated.

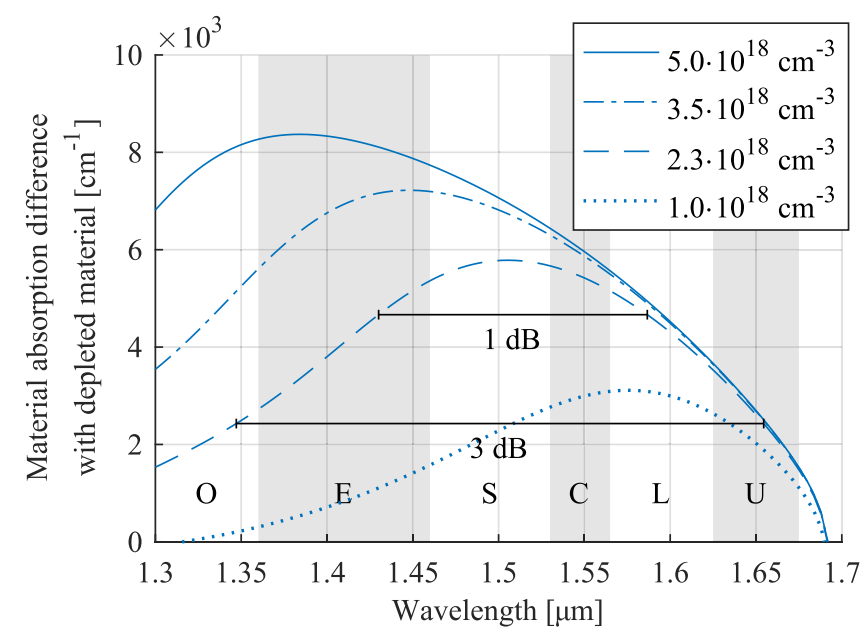

Fig. 3. Absorption in i-InGaAs minus the absorption in n-InGaAs (i.e., the absorption difference that can be achieved for modulation), with telecom bands indicated.

$\Delta \alpha_{\mathrm{m}}\left(E_{\mathrm{p}}, n\right)=\alpha_{\mathrm{m}}\left(E_{\mathrm{p}}, n\right)-\alpha_{\mathrm{m}}\left(E_{\mathrm{p}}, 0\right)$, that can be achieved as a function of wavelength. It is clear that the bandfillin effect is inherently broadband, even at moderately high doping levels and that the broadband nature becomes even more pronounced for higher doping levels.

\section{EAM OPERATION AND DESIGN}

The device is designed to be integrated in the IMOS platform [2]. The platform provides tight confinemen of the optical mode by using a high-index-contrast III-V membrane. The overlap of the optical mode with an absorbing layer can therefore be made high as compared to platforms with low index contrast. As will be discussed later only a thin layer of the material can be absorption modulated. It is therefore essential to design the device in a platform that can achieve a high overlap of this thin layer with the optical mode. The IMOS platform also allows for the double-sided processing that is needed to create the

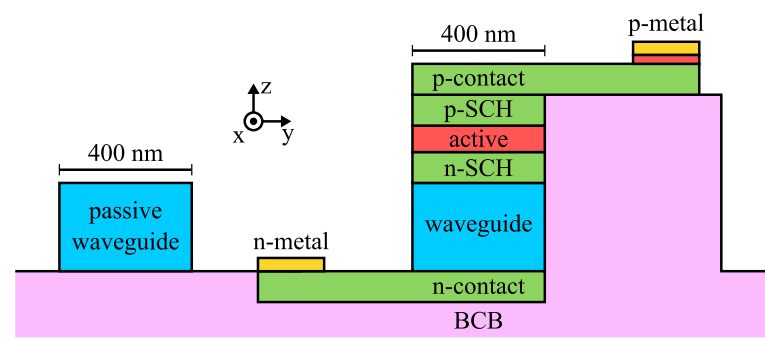

Fig. 4. Cross-section of (left) standard IMOS waveguide, and (right) the simplifie device structure.

TABLE I

LAYERSTACK OF THE ELECTRO-ABSORPTION MODULATOR

\begin{tabular}{lccc}
\hline \hline Purpose & Material & Doping $\left[\mathrm{cm}^{-3}\right]$ & Thickness \\
\hline p-contact layer & p-InGaAs & $1 \cdot 10^{19}$ & $20 \mathrm{~nm}$ \\
p-contact layer & p-InP & $1 \cdot 10^{18}$ & $100 \mathrm{~nm}$ \\
p-SCH layer & -Q1.40 & $1 \cdot 10^{18}$ & $70 \mathrm{~nm}$ \\
p-SCH layer & p-Q1.40 & $3 \cdot 10^{18}$ & $20 \mathrm{~nm}$ \\
Intrinsic buffer & $\mathrm{i}-\mathrm{InP}$ & $\mathrm{n} . \mathrm{i} . \mathrm{d}$. & $10 \mathrm{~nm}$ \\
n-Depletion buffer & $\mathrm{n}-\mathrm{Q} 1.40$ & $3 \cdot 10^{18}$ & $8 \mathrm{~nm}$ \\
Absorption layer & $\mathrm{n}$-InGaAs & $2.3 \cdot 10^{18}$ & $20.5 \mathrm{~nm}$ \\
n-SCH layer & $\mathrm{n}-\mathrm{Q} 1.40$ & $1 \cdot 10^{18}$ & $90 \mathrm{~nm}$ \\
Band-smoothing layer & $\mathrm{n}$-Q1.10 & $5 \cdot 10^{17}$ & $20 \mathrm{~nm}$ \\
Waveguide layer & $\mathrm{i}-\mathrm{InP}$ & $\mathrm{n} . \mathrm{i} . \mathrm{d}$. & $300 \mathrm{~nm}$ \\
n-contact layer & $\mathrm{n}-\mathrm{InP}$ & $2 \cdot 10^{18}$ & $100 \mathrm{~nm}$ \\
\hline \hline
\end{tabular}

structure proposed below [11]. Since IMOS aims for integration of photonics with advanced CMOS electronics, we take the available voltage swing to be $1.5 \mathrm{~V}$.

The mesa of the proposed modulator is based on the standard IMOS waveguide of $300 \mathrm{~nm}$ high and $400 \mathrm{~nm}$ wide. A crosssection of a waveguide and a simplifie cross-section of the modulator are given in Fig. 4. The actual layerstack is given in Table I. The heart of the modulator is formed by the $n$-InGaAs layer above the waveguide. The doping is chosen to be $N_{\mathrm{D}}=$ $2.3 \cdot 10^{18} \mathrm{~cm}^{-3}$, which substantially reduces the absorption of the InGaAs layer, as was discussed in the previous section.

To modulate the electron density in the $n$-InGaAs layer, a p$\mathrm{i}-\mathrm{n}$ junction is created with $\mathrm{i}-\mathrm{InP}$ and $\mathrm{p}-\mathrm{Q} 1.40$ layers. By reverse biasing the junction $\left(V_{\mathrm{a}}=0 \rightarrow-1.5 \mathrm{~V}\right)$, the depletion depth into the n-InGaAs layer increases which leads to higher absorption. The i-InP buffer limits band-to-band tunneling current and increases the avalanche breakdown voltage of the junction.

If the $\mathrm{n}-\mathrm{InGaAs}$ absorption layer is placed directly below the $\mathrm{i}$-InP in the p-i-n junction then the built-in depletion would cause part of the n-InGaAs to be highly absorbing even at $V_{\mathrm{a}}=0 \mathrm{~V}$. Therefore, a n-Q1.40 depletion buffer layer is placed between the $n$-InGaAs absorption layer and the i-InP buffer layer. This reduces the insertion loss. Because the depletion edge is not abrupt, simulation of the modulator in both the optical and semiconductor heterostructure is needed determine the optimal thickness of the n-Q1.40 depletion buffer layer.

The doping levels of the p-Q1.40 SCH layer and n-Q1.40 depletion buffer layer are chosen as high as possible so that the modulation of the depletion depth into the n-InGaAs layer is 


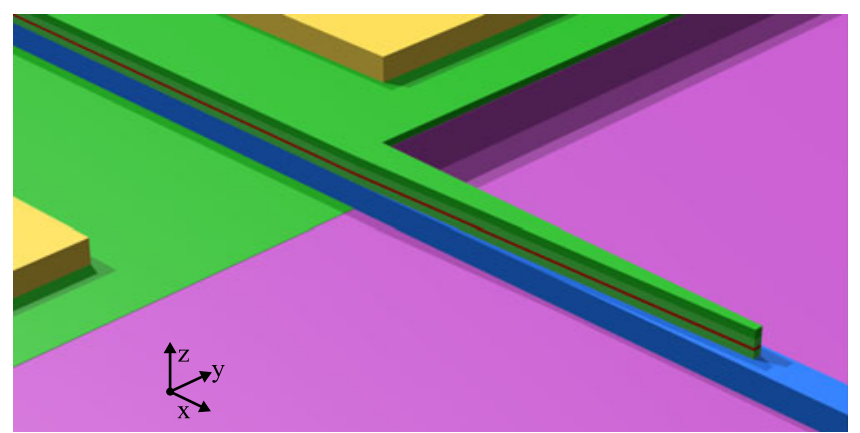

Fig. 5. Visualization of the taper, showing a waveguide (blue) on top of BCB (pink) being tapered into the modulator.

maximized. The doping levels are however limited by the need to avoid excessive band-to-band tunneling current, which can lead to thermal damage [12]. The doping levels in Table I can be achieved in MOCVD epitaxial growth.

Confinemen of the optical mode in the absorption modulated part of the n-InGaAs is improved by the two Q1.40 SCH layers on either side of the $\mathrm{p}-\mathrm{i}-\mathrm{n}$ junction. The refractive index of Q1.40 is higher than InP, which pulls the optical mode up from the InP waveguide layer. In this way the confinemen is increased from $2.01 \%$ to $2.61 \%$, leading to a higher extinction ratio.

A highly doped n-Q1.10 band-smoothing layer is added between the i-InP waveguide and the n-Q1.40 SCH layer to reduce resistance across the junction and the waveguide layer. Because the $\mathrm{i}-\mathrm{InP}$ waveguide layer is located between two n-doped layers, electrons diffuse into the $\mathrm{i}-\mathrm{InP}$ reducing resistance further.

The $\mathrm{p}$ - and $\mathrm{n}$-contact layers are formed by highly doped $\mathrm{p}$ InGaAs, $p$-InP and n-InP layers. Previous results of $\mathrm{Au} / \mathrm{Pt} / \mathrm{Ti}$ metalization for the p-contact and $\mathrm{Au} / \mathrm{Ge} / \mathrm{Ni}$ for the $\mathrm{n}$ contact [13] have shown a specifi contact resistance below $2 \cdot 10^{-6} \Omega \mathrm{cm}^{2}$.

An adiabatic taper was designed to couple light from the waveguide to the device [14]. In this design all the layers above the $\mathrm{i}-\mathrm{InP}$ waveguide layer up to, and including, the p-InP contact layer are tapered along a length of 5 um (see Fig. 5), starting with a width of $100 \mathrm{~nm}$ and ending with the waveguide width of $400 \mathrm{~nm}$. A 3D visualization of the modulator/passive waveguide taper is given in Fig. 5. Increasing the length of the taper further than $5 \mu \mathrm{m}$ does not improve the coupling efficien y (94\%).

For datacom applications an extinction ratio of $7 \mathrm{~dB}$ [15] is desired, as well as a high small signal bandwidth. The electrooptical behavior has been simulated for various thicknesses and doping levels of the n-InGaAs and n-Q1.40 layers. Optimization yields a thickness of $20.5 \mathrm{~nm}$ and $8 \mathrm{~nm}$ for these layers, respectively, and doping levels of $2.3 \cdot 10^{18} \mathrm{~cm}^{-3}$ and $1 \cdot 10^{18} \mathrm{~cm}^{-3}$, respectively (the exact method of optimization is outside of the scope of this paper). To achieve an extinction ratio of $7 \mathrm{~dB}$ the length of the device is chosen to be $80 \mu \mathrm{m}$, excluding tapers.

\section{Simulation OF THE EAM}

To optimize the proposed design the device needs to be simulated accurately. First the numerical simulation and the analytical modeling of the semiconductor physics are discussed. The

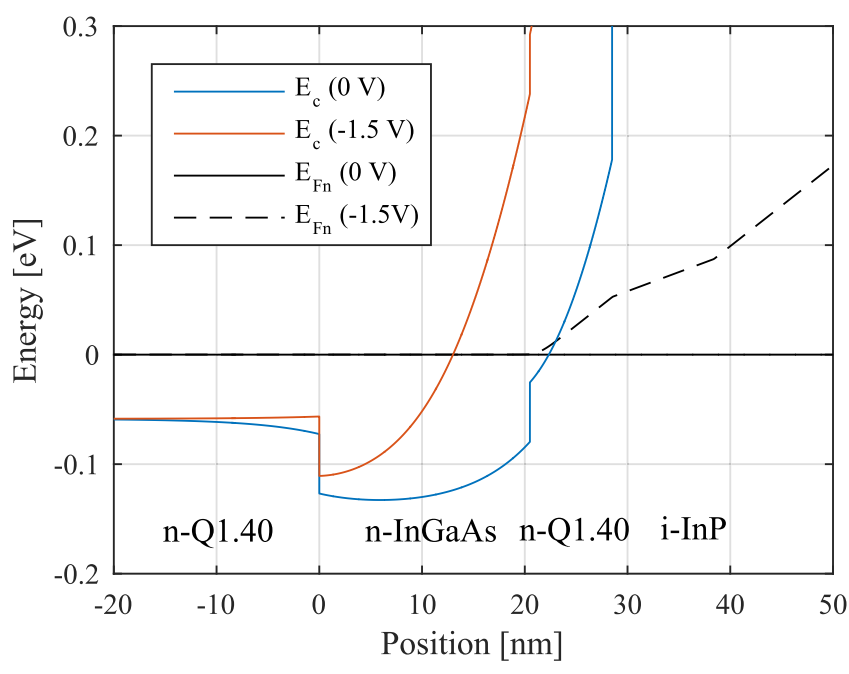

Fig. 6. Conduction band edge around the the n-InGaAs absorption layer for the transparent state $\left(V_{\mathrm{a}}=0 \mathrm{~V}\right)$ and the absorbing state $\left(V_{\mathrm{a}}=-1.5 \mathrm{~V}\right)$.

electron density in the n-InGaAs absorption layer can then be used to determine the absorption profil from the curve following (1). This is used for the simulation in the optical domain with the goal to determine the modal loss as a function of applied voltage.

\section{A. Electron Density Profil From Semiconductor Simulation}

The semiconductor processes are modeled in one dimension (z-axis) because the structure can be considered effectively invariant along the $\mathrm{x}$ - and $\mathrm{y}$-axis (see Fig. 4). The semiconductor simulation includes all layers in Table I. A commercial semiconductor physics simulator [16] is used with band parameters from [17] and carrier mobilities from [18] to determine the band structure and the electron density profil in the n-InGaAs absorption layer.

From the band structure, shown in Fig. 6, it is clear that there will be some quantum confine states in the InGaAs absorption layer due to the narrow thickness of the layer. However, these conduction band states are fille regardless of the applied voltage, as the Fermi level is well above the highest confine quantum state. Because absorbed photons lead to excitation of electrons to empty states around or above the Fermi level, the absorption spectrum of the $\mathrm{n}$-InGaAs layer can be assumed to be identical to that of bulk n-InGaAs. The valence band at $0 \mathrm{~V}$ also gives rise to confine quantum states. However, because of the high Fermi level, no states in the conduction bands are available for these electrons to be excited to by the photons. We can therefore assume that these valence band quantum states have negligible influenc on the absorption spectrum. At $-1.5 \mathrm{~V}$ these states disappear completely.

The electron density profil shown in Fig. 7 makes clear that the depletion edge cannot be approximated as abrupt on these length-scales. Therefore the full depletion approximation cannot be used for an accurate simulation of the device. In this work we use the simulated electron density profil to determine the absorption at every point in the $\mathrm{n}$-InGaAs absorption layer. From the figur the function of the n-Q1.40 depletion buffer is 


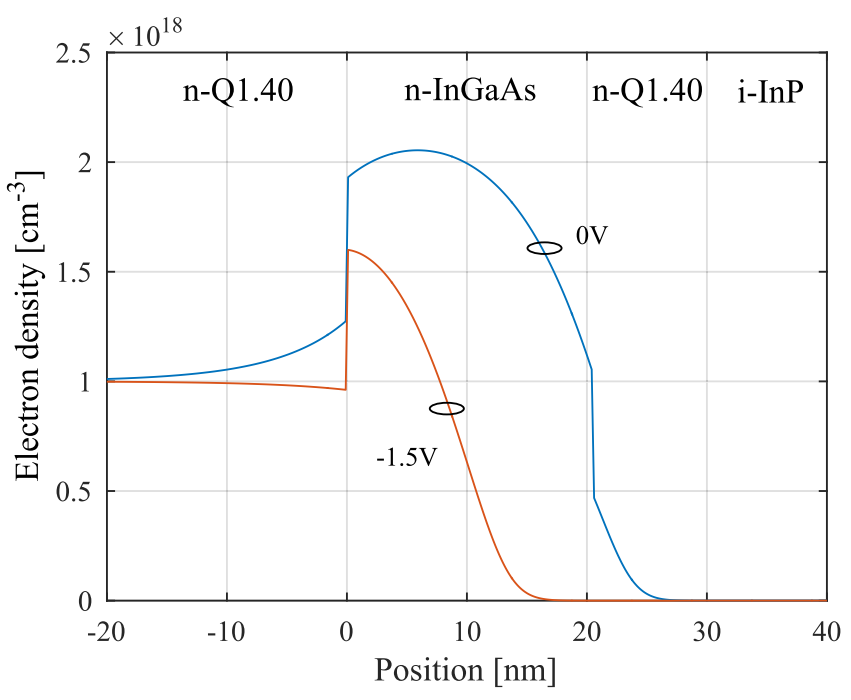

Fig. 7. Electron density in the $\mathrm{n}$-InGaAs absorption layer for the transparent state $\left(V_{\mathrm{a}}=0 \mathrm{~V}\right)$ and the absorbing state $\left(V_{\mathrm{a}}=-1.5 \mathrm{~V}\right.$.)

clear. As this region will always be depleted due to the built-in potential, it would have been highly absorbing at $0 \mathrm{~V}$ had this layer been $\mathrm{n}$-InGaAs. The IL is lowered significantl by this n-Q1.40 depletion buffer layer.

Due to the highly doped layers that form the p-i-n junction the electric fiel across the depletion region is high when a reverse bias is applied. This can lead to avalanche and bandto-band-tunneling currents and the i-InP layer must be thick enough to limit these currents. Using an impact ionization model [19] that includes the dead space effect [20] we estimate the breakdown voltage due to avalanche current to be $-8 \mathrm{~V}$. The band-to-band tunneling current for the proposed modulator is predicted to be well below $3 \mathrm{~mA} \mathrm{[12].} \mathrm{In} \mathrm{the} \mathrm{past} \mathrm{we} \mathrm{have} \mathrm{found}$ that currents higher than $3 \mathrm{~mA}$ caused thermal damage to a uni-traveling-wave photodetector (UTC PD, described in [11]). As the modulator proposed here will have a surface area larger than that of the UTC PD, we can assume that no thermal damage will occur.

\section{B. Modal Loss From Electron Density Profil and Tapering}

The electron density profil is used to determine the refractive index profil (via [9, Fig. 1(b)]) and the material absorption of the n-InGaAs modulation layer (via (1)). Because measurements by Shen et al. [9] do not show material absorption lower than $300 \mathrm{~cm}^{-1}$ we take this as the lowest possible value (shown as the dashed line in Fig. 1). A commercial eigenmode solver [21] is used to fin the modal loss at $1550 \mathrm{~nm}$ as a function of applied voltage. The results are shown in Fig. 8 where we find for a device configuratio with a $20.5 \mathrm{~nm}$ thick $2.3 \cdot 10^{18} \mathrm{~cm}^{-3}$ doped nInGaAs absorption layer, that the predicted IL is $4.9 \mathrm{~dB} / 100 \mathrm{um}$ and the static ER for a $1.5 \mathrm{~V}$ swing is $9.0 \mathrm{~dB} / 100 \mathrm{um}$ at $1550 \mathrm{~nm}$.

The proposed taper is simulated with a commercial 3D finite difference time-domain (FDTD) simulator [21]. The fundamental TE mode inside the device is shown left in Fig. 9. The Q1.40-SCH layers confin the optical mode around the absorption layer. Propagation of the fundamental TE mode through the

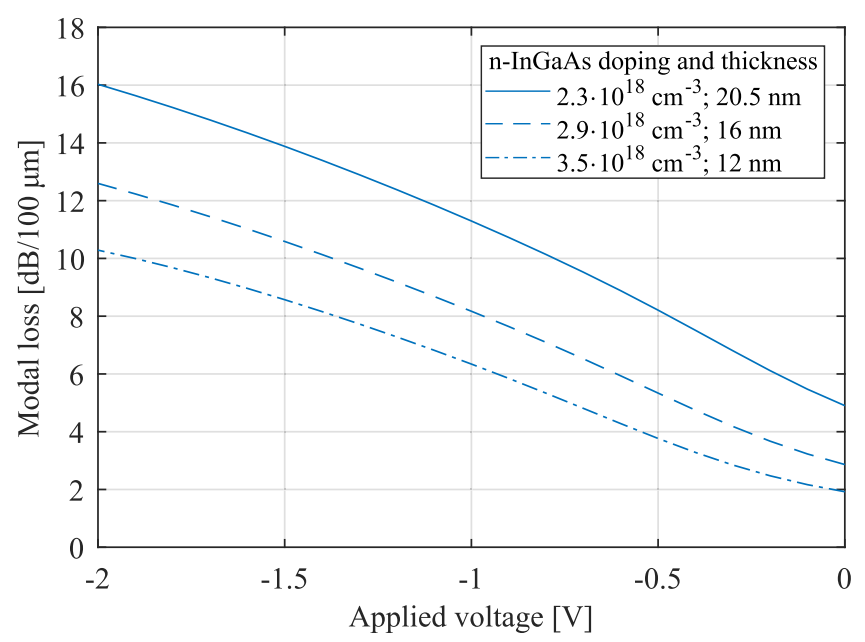

Fig. 8. Modal loss at $1550 \mathrm{~nm}$ of the fundamental TE mode as a function of voltage. The solid line is the device configuratio as discussed in the main text, unless otherwise mentioned.

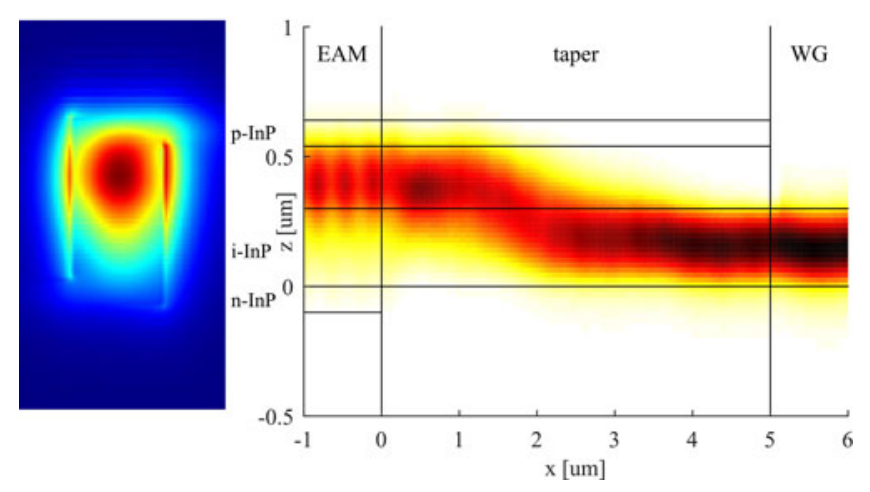

Fig. 9. Fundamental TE mode in the device (left). Propagation (right) of the fundamental TE mode from the EAM into the waveguide through the taper is shown by the electric fiel intensity.

taper is shown right in Fig. 9. Beating due to reflection from the taper are visible, however reflectio back into the fundamental TE mode is less than $1 \%$. Transmission of the fundamental TE mode from the device into the fundamental TE mode of the waveguide is $94 \%(-0.27 \mathrm{~dB})$ [14].

\section{EAM PERformance EVALUATION}

\section{A. Optical Performance}

From the modal loss in Fig. 8 (full line) the ER for a 80 um long device is determined to be $7.2 \mathrm{~dB}$ with an IL of $3.9 \mathrm{~dB}$. Including tapers $(2 \times 0.27 \mathrm{~dB})$ the total IL will be $4.4 \mathrm{~dB}$. The voltage swing was chosen to be $1.5 \mathrm{~V}$, to be compatible with CMOS electronics. If the voltage swing is increased, Fig. 8 shows that the ER increases almost linearly.

Note that the IL can be significantl reduced by compromising the ER as shown by the two dashed lines in Fig. 8. These two configuration only differ by the doping and thickness of the n-InGaAs absorption layer. For the same length of 80 um these achieve an ER of $6.2 \mathrm{~dB}$ and $5.3 \mathrm{~dB}$, with an IL of $2.3 \mathrm{~dB}$ and 
$1.5 \mathrm{~dB}$, respectively. However, to achieve the same 7.2 dB ER a longer, and therefore slower, device is needed.

With increasing temperature the probability distributions in (2) become more spread out, leading to higher absorption that will be most noticable in the transparant state $V_{\mathrm{a}}=0 \mathrm{~V}$. However due to a lack of material parameters, like the constant $C$ in (5), we are not able to predict this change in absorption.

Because the device is operated by modifying the apparent bandgap, the refractive index of $n$-InGaAs will also change with applied voltage. We estimate the refractive index change between the transparent and absorbing state of the InGaAs to be $\Delta n=0.01$ (following [9, Fig. 1(b)]). The phase change is given by $\Delta \Phi=2 \pi \frac{L}{\lambda_{0}} \Gamma \Delta n$. Combined with an overlap of $\Gamma=2.6 \%$ with the depletion modulated part of the $\mathrm{n}$-InGaAs absorption layer, this leads to a phase change of $\Delta \Phi \approx 5^{\circ}$ between the transparent and absorbing state. The linewidth enhancement factor in loss modulators is $\alpha=\Delta n / \Delta k$ [22], where $\Delta n$ and $\Delta k$ are the change in the real and imaginary part of the refractive index, respectively. Taking the change in material absorption $\Delta \alpha_{\mathrm{m}}=5000 \mathrm{~cm}^{-1}$ for the $\mathrm{n}$-InGaAs layer yields $\alpha=0.16$, which is relatively low compared to $\alpha=1-2$ for conventional bulk EAMs, or $\alpha=1.5-1.7$ for directly modulated lasers [22]. This means that the proposed device shows comparatively little frequency chirp.

As was discussed in Section II the bandfillin effect makes for an inherently broadband modulator. To quantify the broadband nature of the device, the wavelength range in which the ER stays within $1 \mathrm{~dB}$ variation is shown in Fig. 3. This range is determined by estimating the ER, for this purpose only, by ER = $4.3 \Delta \alpha_{\mathrm{m}} \Gamma L$, where $\Delta \alpha_{\mathrm{m}}$ is the material absorption difference (yaxis of Fig. 3), $L$ is the device length, and the factor 4.3 converts nepers to decibels. (The ER in other parts of this paper are calculated using a mode solver, as discussed in Section IV-B). We fin that the $1 \mathrm{~dB}$ wavelength range is more than $100 \mathrm{~nm}$. The $3 \mathrm{~dB}$ range is estimated to be over $250 \mathrm{~nm}$, taking into account the absorption of the Q1.40 SCH layers below $1.40 \mathrm{um}$. Replacing these layers with a higher bandgap material will make a $3 \mathrm{~dB}$ range of more than $300 \mathrm{~nm}$ possible.

The variation in IL with wavelength can be estimated in a similar way from Fig. 2. We fin that the variation in IL is larger $(\approx 3 \mathrm{~dB})$ in the range where the ER varies less than $1 \mathrm{~dB}$. However, it should be noted that throughout the $\mathrm{S}, \mathrm{C}$ and $\mathrm{L}$ bands the variation in IL is expected to be less than $2 \mathrm{~dB}$. Furthermore using an $\mathrm{n}$-InGaAs absorption layer with higher doping (like $3.5 \cdot 10^{18} \mathrm{~cm}^{-3}$ ) reduces this variation even more.

Throughout the $\mathrm{S}, \mathrm{C}$ and $\mathrm{L}$ bands the variation in ER and IL is expected to be less than $2 \mathrm{~dB}$. Compared to other EAMs the proposed device is extremely broadband (e.g., $35 \mathrm{~nm}$ for [3] and $80 \mathrm{~nm}$ for [5]). If the $\mathrm{n}$-InGaAs absorption layer is doped to higher levels even more broadband behavior can be expected as shown by the $N_{\mathrm{D}}=5 \cdot 10^{18} \mathrm{~cm}^{-3}$ absorption profile

\section{B. Electrical Performance}

The carrier densities and mobilities resulting from the semiconductor simulation can be used to give an indication of the resistance of the semiconductor layers. The simulation was

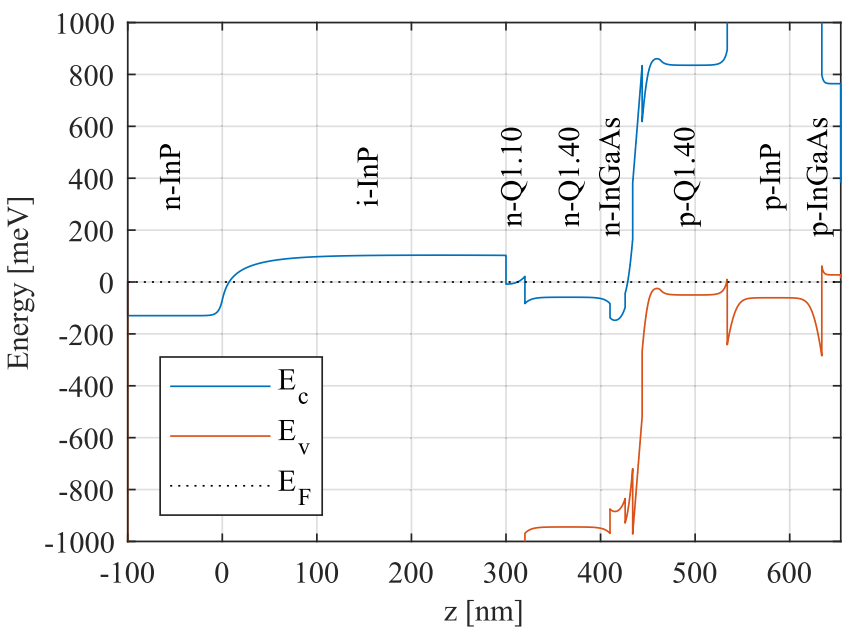

Fig. 10. Band diagram at $0 \mathrm{~V}$ for the layers listed in Table I. Due to lack of space, the labels for the n-Q1.40 depletion buffer layer and the i-InP buffer layer are omitted.

performed in one dimension across all layers listed in Table I. For $0 \mathrm{~V}$ the band diagram is given in Fig. 10. The layers below the $\mathrm{n}$-InGaAs absorption layer are found to give a resistance to the metal contacts of $8 \Omega$, mainly due to the low electron density in the $\mathrm{i}-\mathrm{InP}$ waveguide layer. The removal of generated carriers is aided by the n-Q1.10 band-grading layer (see Fig. 10), and the diffusion of electrons from the n-InP contact layer increase conductivity of the i-InP layer. The p-doped layers above the absorption layer are found to add $35 \Omega$ due to lower mobility of holes. The metal to semiconductor contact resistance is not included in the semiconductor simulation, the metal to semiconductor contact resistance is estimated to increase series resistance by another $10 \Omega$. In total the proposed modulator is thus expected to have a series resistance of $R_{\mathrm{s}}=53 \Omega$.

The junction capacitance $C_{\mathrm{j}}$ can also be extracted from the same semiconductor simulation. It is given by $C_{\mathrm{j}}=\mathrm{d} Q / \mathrm{d} V_{\mathrm{a}}$, where $Q$ is determined by integrating the space charge density over the half-space on one side of the junction. $C_{\mathrm{j}}$ varies between 72 to $115 \mathrm{fF}$ for applied voltages of -1.5 to $0 \mathrm{~V}$ because the depletion depth changes with applied voltage. The simulated junction capacitance matches closely with results from a theoretical full-depletion approximation model. The band-structure and fermi-levels of layers below the $\mathrm{n}$-InGaAs absorption layer do not change between 0 and $-1.5 \mathrm{~V}$ because the depletion region does not extend this far. These layers therefore do not contribute to a change in $Q$ with a change in voltage and do not add to capacitance. Because $C_{\mathrm{j}}$ is proportional to the length of the device, shortening the device will reduce the junction capacitance, but this will also lower the ER.

Taking the average of the junction capacitance leads to an expected cut-off frequency of $f_{c}=1 /\left(2 \pi R_{\mathrm{s}} C_{\mathrm{j}}\right) \approx 30 \mathrm{GHz}$, therefore we estimate a modulation speed above $50 \mathrm{~Gb} / \mathrm{s}$. The modulator is RC limited. The transit-time limit lies well above $100 \mathrm{GHz}$ (determined by the transit of electrons across the i-InP waveguide layer). To increase the modulation speed the doping in the p-contact layer can be increased, as this is a major 
contributor to the resistance. However the increase in freecarrier-absorption would lead to a higher IL.

The power consumption is given in energy-per-bit and estimated from $E_{\mathrm{pb}}=C_{\mathrm{j}} V_{\mathrm{s}}^{2 / 4}=53 \mathrm{fJ} / \mathrm{b}$ [23], with a voltage swing of $V_{\mathrm{s}}=1.5 \mathrm{~V}$. This is higher than $12.8 \mathrm{fJ} / \mathrm{b}$ in [4]. The energy usage is proportional to the junction capacitance, and shortening the device would therefore lower energy usage at the cost of a lower ER.

\section{CONCLUSION}

A novel membrane electro-absorption modulator integrated on silicon and based on band fillin in n-InGaAs is presented. The modulator is predicted to be optically wide-band and to provide an extinction ratio of $7.2 \mathrm{~dB}$, an insertion loss of $4.4 \mathrm{~dB}$, a modulation speed above $50 \mathrm{~Gb} / \mathrm{s}$ and a power consumption of $53 \mathrm{fJ} / \mathrm{b}$. The modulator has a small footprint and operates on the $1.5 \mathrm{~V}$ voltage swing that can be provided by CMOS electronics. This novel modulator is unique in its broadband nature. Using the bandfillin effect in $\mathrm{n}$-InGaAs, it should be possible to achieve record optical bandwidths above $100 \mathrm{~nm}$ while staying within $1 \mathrm{~dB}$ variation of ER. The results of this study show that a promising new EAM is feasible; the basic idea of which can be used in any membrane photonics platform.

\section{REFERENCES}

[1] M. Smit, J. Van der Tol, and M. Hill, "Moore's law in photonics," Laser Photon. Rev., vol. 6, no. 1, pp. 1-13, 2012.

[2] J. van der Tol et al., "Photonic integration in indium-phosphide membranes on silicon (IMOS)," in Proc. SPIE 8988, Integr. Opt., Devices, Mater., Technol. XVIII, 2014, pp. 89880M-1-89880M-17.

[3] S. Gupta et al., " $50 \mathrm{GHz}$ Ge waveguide electro-absorption modulator integrated in a $220 \mathrm{~nm}$ SOI photonics platform," in Proc. Opt. Fiber Commun. Conf. Exhib., Mar. 2015, Paper Tu2A.4.

[4] S. A. Srinivasan et al., " $56 \mathrm{~Gb} / \mathrm{s}$ germanium waveguide electro-absorption modulator," J. Lightw. Technol., vol. 34, no. 2, pp. 419-424, Jan. 2016.

[5] Y. Hu et al., "Broadband 10Gb/s graphene electro-absorption modulator on silicon for chip-level optical interconnects," in Proc. IEEE Int. Electron Devices Meeting, 2014, pp. 5.6.1-5.6.4.

[6] A. Millán-Mejia, J. van der Tol, and M. Smit, "Design and simulation of a high bandwidth optical modulator for IMOS technology based on slot-waveguide with electro optical polymer," in Proc. 18th Annu. Symp. IEEE Photon. Benelux Ch., 2013, pp. 195-198.

[7] D. Hahn, O. Jaschinski, H.-H. Wehmann, A. Schlachetzki, and M. Von Ortenberg, "Electron-concentration dependence of absorption and refraction in n-In0.53Ga0.47As near the band-edge," J. Electron. Mater., vol. 24, no. 10, pp. 1357-1361, 1995.

[8] B. R. Bennett et al., "Carrier-induced change in refractive index of InP, GaAs and InGaAsP," IEEE J. Quantum Electron., vol. 26, no. 1, pp. $113-$ 122, Jan. 1990

[9] L. Shen et al., "Ohmic contacts with ultra-low optical loss on heavily doped n-type InGaAs and InGaAsP for InP-based photonic membranes," IEEE Photon. J., vol. 8, no. 1, Feb. 2016, Art. no. 4500210.

[10] J.-P. Weber, "Optimization of the carrier-induced effective index change in InGaAsP waveguides-application to tunable Bragg filters" IEEE $J$. Quantum Electron., vol. 30, no. 8, pp. 1801-1816, Aug. 1994.

[11] L. Shen et al., "High-bandwidth uni-traveling carrier waveguide photodetector on an InP-membrane-on-silicon platform," Optics Express, vol. 24, no. 8, pp. 8290-8301, 2016.

[12] J. van Engelen, L. Shen, J. van der Tol, and M. Smit, "Modelling band-toband tunneling current in InP-based heterostructure photonic devices," in Proc. 20th Annu. Symp. IEEE Photon. Benelux Ch., 2015, pp. 27-30.

[13] L. Shen et al., "Low-optical-loss, low-resistance Ag/Ge based ohmic contacts to n-type InP for membrane based waveguide devices," Opt. Mater. Express, vol. 5, no. 2, pp. 393-398, 2015.
[14] J. van Engelen et al., "Optical design of a novel wide-band membrane electro-absorption modulator based on bandfilling" in Proc. 21st Annu. Symp. IEEE Photon. Soc. Benelux Ch., 2016, pp. 227-230.

[15] G. T. Reed, G. Mashanovich, F. Gardes, and D. Thomson, "Silicon optical modulators," Nature Photon., vol. 4, no. 8, pp. 518-526, 2010.

[16] Nextnano GmbH., 2017. [Online]. Available: http://www.nextnano.com/ nextnanoplus/index.htm

[17] I. Vurgaftman, J. Meyer, and L. Ram-Mohan, "Band parameters for III-V compound semiconductors and their alloys," J. Appl. Phys., vol. 89, no. 11, pp. 5815-5875, 2001.

[18] M. Sotoodeh, A. Khalid, and A. Rezazadeh, "Empirical low-fiel mobility model for III-V compounds applicable in device simulation codes," $J$ Appl. Phys., vol. 87, no. 6, pp. 2890-2900, 2000.

[19] Y. Okuto and C. Crowell, "Ionization coefficient in semiconductors: A nonlocalized property," Phys. Rev. B, vol. 10, no. 10, 1974, Art. no. 4284.

[20] P. Bhattacharya, Semiconductor Optoelectronic Devices. Englewood Cliffs, NJ, USA: Prentice-Hall, 1994.

[21] Lumerical Solutions Inc., 2017. [Online]. Available: https://www. lumerical.com/tcad-products/

[22] F. Koyama and K. Iga, "Frequency chirping in external modulators," $J$. Lightw. Technol., vol. LT-6, no. 1, pp. 87-93, Jan. 1988.

[23] G. T. Reed et al., "Recent breakthroughs in carrier depletion based silicon optical modulators," Nanophotonics, vol. 3, no. 4/5, pp. 229-245, 2014.

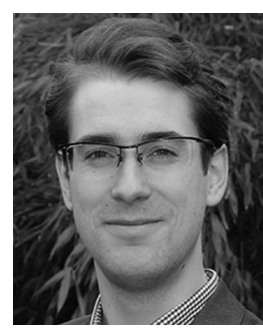

Jorn P. van Engelen received the M.Sc. degree in electrical engineering from Eindhoven University of Technology, Eindhoven, The Netherlands, in 2016, where he is currently working toward the Ph.D. degree at the Institute for Photonic Integration. His current research interests include the novel bandfilling-base electro-absorption modulator, bringing the $\mathrm{AlGaInAs}$ material system to the IMOS platform, and the integration of actives and passives in the IMOS platform.

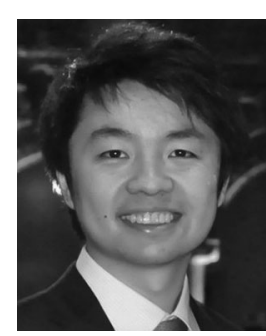

Longfei Shen was born in Beijing, China, in 1988. He received the Bachelor's degree in electrical engineering from the Zhejiang University, Hangzhou, China, in 2010. He subsequently moved to Europe to study photonics at the Royal Institute of Technology (KTH), Stockholm, Sweden, supported by the Erasmus Mundus scholarship. He received the Master's degree in 2012, with a thesis project performed at Philips Research and the Ph.D. degree (cum laude) from the Eindhoven University of Technology, Eindhoven, The Netherlands, based on research in highspeed photodetectors and heterogeneous integration technology. He is currently with the JePPIX Technology Center, working on photonics technology development and transfer from academic research to industrial applications.

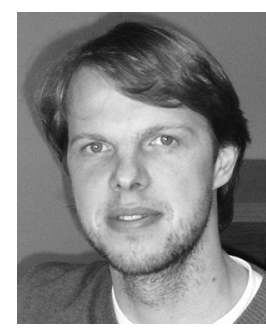

Gunther Roelkens received a degree in electrical engineering from Ghent University, Belgium, in 2002 and the Ph.D. degree from the same university in 2007, at the Department of Information Technology (INTEC), where he is currently an Associate Professor. In 2008, he was a Visiting Scientist in IBM TJ Watson Research Center, New York, NY, USA. His research interest includes the heterogeneous integration of III-V semiconductors and other materials on top of silicon waveguide circuits and electronic/photonic cointegration. He was holder of an ERC starting grant (MIRACLE), to start up research in the fiel of integrated mid-infrared photonic integrated circuits. 


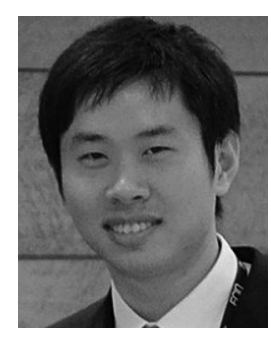

Yuqing Jiao was born in Hangzhou, China. He received the Ph.D. degrees from both Eindhoven University of Technology, the Netherlands, and Zhejiang University, Hangzhou, China, in 2013. Since then he continued his research at Eindhoven University of Technology. Since 2016, he is appointed as an Assistant Professor at the Institute of Photonic Integration (IPI, former COBRA Research Institute), Eindhoven University of Technology. His research topic is focused on a novel III-V-based nanophotonic platform. $\mathrm{He}$ is focusing on ultrafast and strong light-matter interactions in submicron optical confinement Applications span from optical interconnects, ultrafast photonic devices to optical beam steering and optical sensing. He has strong background and expertise in a wide range of photonic materials (from silicon to III-V) and nanotechnologies. He has (co-)authored more than 20 international journal publications and 50 conference papers. He is a member of the IEEE Photonics Society and the Optical Society of America. Currently, he serves as a board member of IEEE Photonics Society Benelux Chapter.

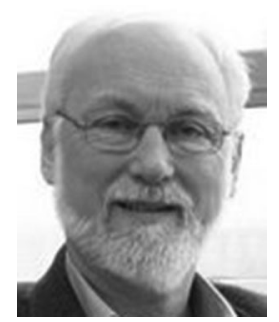

Meint K. Smit received the Graduate degree in electrical engineering from the Delft University of Technology, the Netherlands, in 1974 and the Ph.D. degree in 1991, both with honours. He was closely involved in the introduction of MMI-couplers in semiconductor-based Photonic IC technology. From 2000 to 2014, he was the Chair of the Photonic Integration group, COBRA Research Institute, Eindhoven University of Technology. His current research interests include InP-based photonic integration, including integration of InP circuitry on Silicon. He is the founder of the JePPIX platform, the Joint European Platform for Photonic Integration of Components and Circuits, and strongly involved in the development of the InP-based photonic foundry system in Europe. He started research in integrated optics in 1981 and is inventor of the Arrayed Waveguide Grating, for which he received a LEOS Technical Achievement award in 1997 and the Rank Prize in 2016. He is an IEEE Photonics Society Fellow and received an ERC Advanced Grant in 2012.

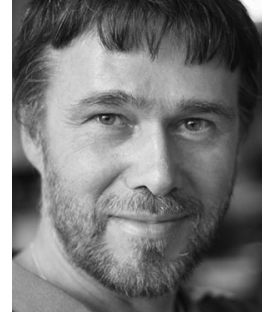

Jos J. G. M. van der Tol received the M.Sc. and Ph.D. degrees in physics from the State University of Leiden, Leiden, The Netherlands, in 1979 and 1985, respectively. In 1985, he joined KPN Research, where he became involved in research on integrated optical components for use in telecommunication networks. His research interest in this fiel have covered modeling of waveguides, design of electro-optical devices on lithium niobate and their fabrication. Furthermore, he has been working on guided wave components on III-V semiconductor materials. He has also been active in the fiel of optical networks, focussing on survivability, introduction scenarios, and management issues. Since July 1999, He is working as an Associate Professor at the University of Technology Eindhoven, The Netherlands, where his research interests include opto-electronic integration, polarization issues, photonic membranes, and photonic crystals. He is (co-)author of more than 160 Publications in the field of integrated optics and optical networks, and has 25 patent applications to his name. 